\title{
Study on the Evolution Mechanism of Island Tourism System from the Perspective of Dissipative Structure-In Light of Chuanshan Archipelago
}

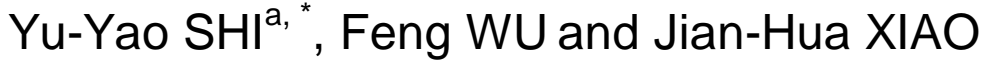

School of Economics and Management, Wuyi University, Jiangmen, Guangdong 529020, China

a351118381@qq.com

*Corresponding author

Keywords: Theory of Dissipative Structure, Chuanshan Archipelago, Evolution Process, Evolutionary Mechanism.

\begin{abstract}
In today's world, islands have been hot spots for global tourists due to their unique natural landscapes and cultural environments. Thus, how to develop the island tourism sustainably becomes a worthy question to explore. So far, few studies discuss the island tourism from the perspective of system science. Based on the theory of dissipative structure, this paper analyzes the evolution and its dynamic mechanism of Chuanshan Archipelago tourism system with dividing the process into four stages. It finds that the evolutionary impetus comes from the nonlinear action and the coupling relationship among various elements of the self-organization and heter-organization in tourism system. During the course of evolution, the tourism system constantly fluctuates. Therefore, in order to avoid a path of decline, the nonlinear operating mechanism should be improved to constantly obtain a negative entropy and promote an order development of tourism system.
\end{abstract}

\section{Introduction}

The study of tourist destination has always been one of the hot topics in tourism study. Meanwhile, analyzing evolution characteristics of the tourism system is conductive to guide an orderly and coordinated development of tourism. The traditional evolution theory $-\mathrm{R}$. W. Butler's Tourist Area Cycle of Evolution has been widely used in different tourism destinations such as mountains, islands, caves and theme parks, which provides a clear understanding on the evolution law, operational mechanism, characteristics and influencing factors of these sites. Nevertheless, Butler's theoretical model is not applicable to explain many in-depth issues, such as the development mode, product transition, functional promotion of tourism and changes from related social support system. Besides, the division of each life cycle in this theory is qualitative and arbitrariness also remains[1].

Dissipative structure theory explains how an open system operates from disordered to ordered state, and many domestic researchers have applied this theory into their tourism-related research fields. Wang first proposed the concept of Tourism Dissipative Structure System (TDSS) in 2006. Four years later, Lu and Bao analyzed the evolution of Qiandao lake tourism destination and found that it was a dissipative structure system supported by the external natural and social-economic environment, including the attraction subsystem, the infrastructure subsystem, the service subsystem and the support subsystem. They eventually drew the conclusion that the essence of evolution was a negative entropy input process of the dissipative structure and the impetus of evolution was the nonlinear interaction of system elements. Overall, it is an important issue for tourism studies to discuss the evolution of tourism destination system, grasp the tendency of system energy dissipation, explore the vital force to drive system evolution, and promote the evolution to an orderly state[2].

\section{Data source and Research Method}

Because of the unique natural environment and cultural environment, islands have increasingly become popular destinations for tourists. Hence, the sustainable development of island tourism is worthy to be discussed. As the second largest archipelago in China, Chuanshan Archipelago covers an area of 
approximately 240 square kilometers. The local government, based on advantaged geographical location and profound historical and cultural connotation, regards tourism as the pillar industry with relatively typical evolution of the dissipative structure system. In light of the development process of tourism in Chuanshan Archipelago, this paper will elaborate evolution mechanism of the dissipative structure in island tourism.

In order to ensure the consistency of statistical caliber and the integrity of the data, the Jiangmen Statistical Yearbook and the Taishan Statistical Yearbook are two main official data sources in this research, including the annual number of tourists, natural, social and economic development and other related information of Chuanshan Archipelago. This paper focuses on years from 1980 to 2014, and according to the tourist flow within 34 years, the evolution of Chuanshan Archipelago is carefully divided into four stages in chronological order. By virtue of the dissipative structure theory, the periodic characteristics of the evolution are elaborated; the major problems in different periods are extracted; and the optimization measures are summarized. In general, through the combination of qualitative and qualitative methods, this paper aims to critically analyze the evolution of tourism dissipative system and promote the scientific development of tourism in Chuanshan Archipelago.

\section{Analysis of the Evolution of Tourism System in Chuanshan Archipelago}

The formation condition of dissipative structure is firstly an open system. Under the condition of an open system, the entropy of the system consists of the entropy exchange between the system and the outside world and the entropy inside the system. When the entropy exchange between the system and the outside world is less than zero, and if the negative entropy flow is sufficiently strong, the total entropy of the system will decrease, so that the system will develop toward an orderly state[3].

Therefore, the evolution of tourism dissipative structure system is the generation process of the system from disorder to order. Considering the degree of orderly change of the dissipative structure system, and taking into account the interaction of various elements within the system, the process of Chuanshan Archipelago tourism system evolution can be roughly divided into four stages, which are the embryonic stage, the formation stage, the development stage and the optimization stage.

\section{Embryonic Stage}

With its beautiful scenery and unique natural resources, the tourism industry firstly began to develop in Shangchuan Island and Xiachuan Island. After 1980, some government departments and university student organizations came to Shangchuan Island for social investigations and internships. Under the propaganda of word-of-mouth, the reputation of Shangchuan Island gradually formed and the tourism of Shangchuan Island began to sprout.

With the rising popularity of Shangchuan Island, the Beach Tourism Center was organized by the Shangchuan Office in 1983. From 1985 to 1991, the infrastructure construction of the tourism hotel in Shangchuan Island was accelerated, and Haiyue building, Haitao villa and Guanri building were built. In 1990, the attractions of the scenic area were gradually enriched. Under the investment of Hong Kong businessmen, the beach became the first scenic spot with water parachutes in China.

Xiachuan Island started its tourism later than Shangchuan Island. In 1984, Xiachuan Island set up a preparatory team of "Wangfuzhou Sea Bath Tourism Center" to raise funds of 120,000 and built "Sifang Building", and "Little Stone House" etc. In 1985, Xiachuan tourism company was established, and the "Wangfuzhou tourism center" began to test the industry. It received 16,000 visitors and the tourist area reception capacity was not enough.

The tourist industry of Chuanshan Archipelago started to sprout in the 80s of last century. In its initial stage, the infrastructure of tourist facilities in Shangchuan and Xiachuan Island was relatively primitive and the reception capacity was obviously insufficient. At that time, the number and scale of tourists were small, and the economic and social benefits of tourism were not obvious, and the tourism system was not formed yet.

\section{Formation Stage}

In 1992, Chuanshan Archipelago was listed as "Comprehensive Experimental Zone for Tourism Development in Guangdong Province". Meanwhile, "Shangchuan Island Beach Tourist Zone" and 
"Xiachuan Island Wangfuzhou Tourist Zone" were designated as provincial tourist resorts, which promoted the tourism of Chuanshan Archipelago. There are more than 6000 guest rooms provided by the hotel and two villas, more than 20 scenic spots, and the tourist area is equipped with more than 20 entertainment projects in the Beach and Wangfuzhou. With a total investment of more than 2 billion yuan in various construction projects in Shangchuan and Xiachuan Island, the infrastructure and supporting facilities had been greatly improved.

In 1992, Guangdong provincial government established Chuanshan Archipelago as a "comprehensive experimental zone for tourism development", which resulted in the birth of the earliest tourism administration department of Chuanshan Archipelago. Subsequently, the jurisdiction of Chuanshan Archipelago was adjusted respectively in 2001 and 2003, and the towns of Shangchuan and Xiachuan were withdrawn and merged into the town of Chuanshan Archipelago. There were 9 agencies under the town of Chuanshan Archipelago, which were responsible for the management of tourism construction and the regular work of the government, forming a tourism management mode led by the government.

In 1993, the beach tourist area set up a "Shangchuan People's Government Beach Tourism Administration Office" to be responsible for all aspects of scenic management. In 1994, the Beach Tourism Center was established, which is the first member of the Guangdong tourism association. At first, the two parts were independently operated, but merged in 1996, and renamed as the "Beach Tourism Administration" in 2000, which was under the jurisdiction of the township government of Chuanshan Archipelago in 2001.

Chuanshan Archipelago tourism system has gradually formed, and the tourist area environment has been improved, attracting more and more tourists. In 2004, Chuanshan Archipelago received 900,000 tourists, with 350 million yuan of the tourism revenue. With more than 40,000 visitors on Labor Day, tourism has become a pillar industry in Chuanshan Archipelago.

\section{Development Stage}

Chuanshan Archipelago has never stopped the pace of development, facing the good momentum of tourism development, and constantly improved its tourist area. In 2009, Chuanshan Archipelago was rated as a 4A-level tourist attraction by the National Tourism Administration, making the development of tourism a higher level. Since the development of tourism in Chuanshan Archipelago Town, the tourism revenue increased year by year. Total tourism revenue grew fastest in 2009, compared with the previous year increased by $33 \%$. In 2012, the total tourism revenue exceeded 500 million yuan. In 2013, the tourism revenue was 1.9 times than that of 2007 , reaching 570 million yuan. The overall tourism industry in Chuanshan Archipelago Town was in a good state of development.

In 2008, Chuanshan Archipelago Tourism Area successively won the honorary titles of "Guangdong Province Tourist Town" and so on, but the total tourism revenue accounted for only $39.72 \%$ of GDP, and the leading role of tourism industry is not prominent. In 2010, focusing on the development of tourism as a leading industry, it won the first title of "coastal tourism demonstration zone of Guangdong Province" and the tourism industry was thriving.

In 2011, Chuanshan Archipelago grasped the infrastructure construction of tourism industry and actively participated in the "2011 China (Guangdong) International Tourism Industry Fair and "The 6th Cross-Strait Tourism Exhibition (Taipei)". In 2012, the town changed its economic development mode, and the momentum of tourism development continued to be strong. It ushered in a new round of tourism investment boom, and many new hotels were launched, and the facilities on the island were gradually improved. At present, Chuanshan Archipelago has formed a service industry chain integrating "eating, living, entertaining, shopping and traveling" after many years of development.

\section{Optimization Stage}

During the optimization period, each subsystem is further improved, and the coordination between subsystems has led to the development of the overall effect of the tourism system in the optimal direction. The external environment of the system has changed, and the state has paid more and more attention to the tourism industry. The introduction of the series of policies provides a favorable environment for the development of Chuanshan Archipelago tourism. The internal structure of demand subsystem is also gradually changing. In terms of demand quantity, with the rapid growth of national economy and the 
change of holiday system, the domestic tourism demand has been greatly improved, and the scope of the tourism market has been greatly expanded. In terms of demand type, the tourism is gradually transformed from sightseeing to leisure, and the demand level and demand category are also diversified.

In 2014, Shangchuan Island and Xiachuan Island were rated as the top ten beautiful islands in Guangdong Province. In 2015, Shangchuan Island and Xiachuan Island advanced to the top 20 of "China's Beautiful Island". However, the tourism economic benefits of Chuanshan Archipelago in Jiangmen city are not fully developed, and further development is the direction that Chuanshan Archipelago needs to make efforts. Therefore, Chuanshan Archipelago was clearly defined in the general plan for tourism development in Jiangmen (2013-2025), the policy stipulates the construction of Jiangmen Binhai tourism brand through Chuanshan Archipelago and the nearby coastal tourism group. After nearly 40 years of exploration and development, the tourist facilities in Hainan Island have been gradually improved and become the national 4A-level comprehensive tourist resort with strong reception capacity.

Over the past 40 years, the evolution of Chuanshan Archipelago has undergone four periods of development. The dissipative structure of the system has experienced from the initial disordered development to low orderly, and then from low order to high order transition, and finally toward the high order process. In the process of the sequential increase of the system, the tourism industry system is gradually formed, and the spatial structure is gradually optimized, and the function of the tourism system is gradually improved.

\section{Evolutionary Mechanism of Chuanshan Archipelago Tourism System}

\section{The Essence of Evolution: Enter Negative Entropy into the Dissipative Structural System}

The essence of Chuanshan Archipelago tourism system evolution is the result of negative entropy input continuously. When the degree of system opening is large enough, the entropy exchange deS is negative entropy, and the relation $|\mathrm{deS}|>$ diS is satisfied, the system can self-organize. According to Wang Diyun's summary, the negative entropy of tourist destinations mainly includes the following sources: nature, society, tourism development and tourism operation.

\section{The Power of Evolution: The non-linear Effect of System Elements}

Let system characteristics be described as differentiable functions $y=f(x), x_{0}$ is the system equilibrium state, $\Delta \mathrm{x}$ is the deviation from the equilibrium state, The effect of $\Delta \mathrm{x}$ on the performance of the system is nonlinear, In the near equilibrium state $\mathrm{f}(\mathrm{x})$ by the Taylor series expansion, Obtained: $\mathrm{y}=\mathrm{f}(\mathrm{x}+\Delta \mathrm{x})=\mathrm{a}+\mathrm{b} \Delta \mathrm{x}+\mathrm{h}(\Delta \mathrm{x})$.

When $\Delta \mathrm{x}$ is small enough (the system is in an equilibrium state), the nonlinear effect $\mathrm{h} \Delta \mathrm{x}$ is a negligible disturbance, $y$ is determined by the linear term $b \Delta x$ and the system has a near-equilibrium structure, at this time it's not possible to produce a self-organizing structure. However, as $\Delta x$ increases, the effect of nonlinear factors also increases. Once it increases sufficiently large that the influence of linear term $b \Delta x$ will be reduced to a secondary position as the critical point arrives far away from the equilibrium state, the system properties mainly consist of Nonlinear term $\mathrm{h} \Delta \mathrm{x}$ to decide, the system will self-organization dissipative structure.

This process works through the self-organization of the system, at the same time, it also relies on the heter-organization effect as the mainstay of external constraints. Therefore, the emergence of the system of dissipative structure in tourist destinations is the result of the combination of self-organization and its organizational role. Through the "fluctuation" movement of the system under the non-linear effect of various factors, it promotes the formation of new ordered structures. When "fluctuation" continues to increase, the state of system elements changes while a certain threshold is crossed. When the system finally reaches a state of "huge fluctuation", the original order gradually disintegrates and a new orderly structure gradually emerges, then the tourism system will evolve to a new stage. The evolution of the tourist destination of the Chuanshan Archipelago is also the result of the common promotion of the tourist destination system under the non-linear action of various elements (Fig. 1). 


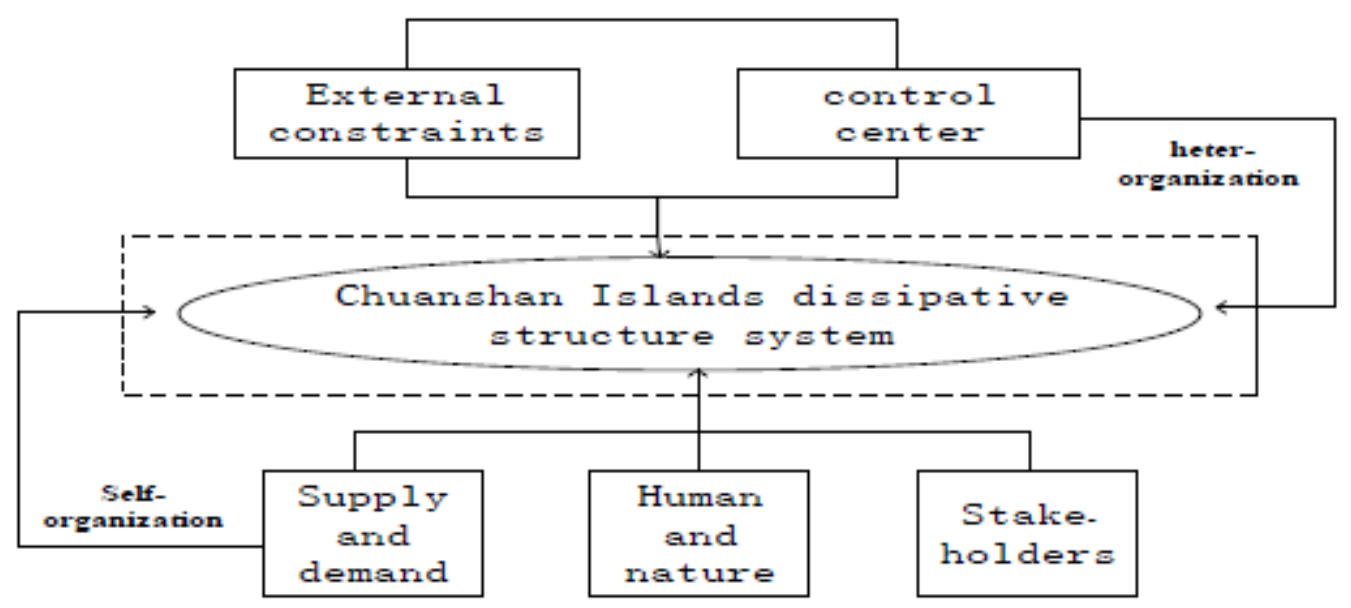

Fig. 1 The evolution mechanism of Chuanshan archipelago

\section{Heter-organization of Tourism System.}

(1)Heter-organization of External environment. Any system generated from weak to strong, or from one aspect into another form, they are inseparable from the influence and effect on the environment. In 2004, CGNPC invested in wind power generation project in Chuanshan Archipelago. The project was included in the wind power construction project of "Eleventh Five-Year Plan of Energy Development in Guangdong Province". The electricity generated by wind power can be reduced by 13.75 tons of carbon dioxide emissions, which is of great significance to the protection and improvement of the natural environment. Chuanshan Archipelago is located in Jiangmen City of the South China Sea in the South China Sea, which is the "China's First Overseas Hometown". Adjacent to Hong Kong and Macau, the tourism resources are rich and the environment is beautiful, where has collection of towers, islands, hot springs, hometown culture and many other travel topics.

(2)Heter-organization of control center. The system tends to evolve in a centralized way during the evolutionary process[4]. Individual components gradually evolve and gain the domination of other components, then becoming the administrator of the entire system. The government assumed the function of a control center and conducted its organizational activities of organizing, directing, controlling and governing other components in the evolution of Chuanshan Archipelago.

Self-organization of Tourism System. The non-linear interaction within the system is its self-organizing force. The evolution of the tourism system in Chuanshan Islands is propelled by the three basic non-linear relations.

(1) The non-linear effect of supply and demand. The Economic and social environment of Chuanshan Archipelago is very well. In addition, the state had adjusted the system of national vacation, the vacation has become an important choice for the public to travel. And people's travel demand tends to be diversified and quality-oriented. Chuanshan Archipelago complied with the development requirements of the times and the increase in supply also stimulated more demand. People's awareness of Chuanshan Archipelago tourism and the willingness to travel increased.

(2) The non-linear effect of human-nature relationship. Chuanshan Archipelago changed in response to people's needs, focusing on the impact of project development based on the increase number in tourists and their preferences. Chuanshan Archipelago offer more than 20 tourism projects to meet the various needs of tourists at different levels of consumption. As continuing to tilt people's needs, the capacity of the region is challenged. The coordination between people and land is gradually being broken, and the negative effects of pursuing the maximization of interests begin to emerge. For the sustainable development of tourism, Chuanshan Archipelago took measures to maintain a good ecological environment and promote the sustainable development of human and nature.

(3) The non-linear role of the various stakeholders. In order to fully mobilize the enthusiasm of all parties and promote the harmonious development of tourism destinations, we must ensure various stakeholders can benefit from tourism development. Through improving the tourism management system, 
Chuanshan Archipelago tourist area has given full play to the subjective initiative of the four main bodies, namely, the government, tourists, tourism enterprises and tourism destination residents.

\section{Conclusion and Suggestion}

The tourist destination system of Chuanshan Archipelago is a dissipative structure system based on tourists, which constantly exchanges material and surroundings with the surrounding environment so that the internal system can be transformed into an orderly state from disorderedness. It is a multi-functional and multi-level complex composed of nature, society, economy and politics. People through social activities of production and living to create, transform and expand the dissipating system of tourist destination as same as the formation, development, prosperity, decline of the destination dissipative system. The succession of the tourist destination system of the Chuanshan Archipelago has undergone four phase of embryonic stage, formation stage, development stage and optimization stage. According to the dissipative structure theory, we must continuously input the negative entropy flow to the system to avoid decay. Through intervening the dissipative system of tourist destination, such as introduction of new theories, management mode innovation, laws' and regulations' adjustment, it can improve the degree of self-organization and order of Chuanshan Archipelago tourism system, which in turn can enhance material, energy, information processing and conversion capabilities of system, and ultimately create a destination dissipation system with beautiful environment, reasonable structure, strong stability, high production efficiency[5].

The destination dissipation system of Chuanshan Archipelago is an open system with feedback regulation. If we pay too much attention to the benefits derived from tourism but neglect the negative effects, the total entropy of the tourism dissipation system in Chuanshan Archipelago will increase drastically, causing the system deviation to aggravate and unstable. May even prompt the destination into the decline as soon as possible. Only by perfecting the system of non-linear development mechanism of system destination and carrying out scientific tourism construction, the negative entropy flow would be introduced from external system as much as possible under the constraints of the natural geographical resources of tourism destination. System achieves a greater degree of matter and energy dissipation, making an orderly spatial, temporal and functional structure to obtain the best overall efficiency.

\section{Acknowledgement}

This research was financially supported by the Universities in Guangdong Province features innovative projects "Research on Oceanic Industry Investigation and Development of Da Guang Hai Bay" (Grant No.2016WTSCX114).

\section{References}

[1] Tang L, Jang S. The Evolution from Transportation to Tourism: The Case of the New York Canal System[J]. Tourism Geographies, 2010, 12(3): 435-459.

[2] Țigu G, Calaretu B. Supply Chain Management Performance in Tourism. Continental Hotels Chain Case[J]. Amfiteatru Economic, 2013, 15(33): 103-115.

[3] Weng J, Yang K. Spatial Structure of Tourism System: Spatial Model for Monopolistic Competition with Asymmetry[J]. Systems Engineering - Theory \& Practice, 2007, 27(2): 76-82.

[4] Smith W W. The Origins of Hospitality and Tourism[J]. Annals of Tourism Research, 2011, 38(4): 1666-1667.

[5] Dimmock K, Musa G. Scuba diving tourism system: a framework for collaborative management and sustainability[J]. Marine Policy, 2015: 52-58. 